\title{
ARTICLE \\ Composition of Prosobranchia-Pulmonata (Mollusca: Gastropoda) in rocky intertidal zone in the Marine Priority Region 32, Guerrero, Mexico
}

\author{
Composición de Prosobranchia-Pulmonata (Mollusca:Gastropoda), asociada a la zona \\ intermareal rocosa en la Región Marina Prioritaria 32, Guerrero, México

\section{Carmina Torreblanca-Ramírez ${ }^{1}$, Rafael Flores-Garza ${ }^{2}$, Pedro Flores-Rodríguez ${ }^{2}$, Sergio García-Ibáñez ${ }^{2}$, Jesús Emilio Michel-Morfín, José Luis Rosas-Acevedo ${ }^{1}$ and Arcadio Valdés-González ${ }^{4}$}

\begin{abstract}
${ }^{1}$ Unidad de Ciencias de Desarrollo Regional, Universidad Autónoma de Guerrero, Calle Pino s/n Colonia El Roble, C.P. 39640 Acapulco, Guerrero, México. carminatorreblanca@yahoo.com.mx

${ }^{2}$ Facultad de Ecología Marina, Universidad Autónoma de Guerrero, Av. Gran Vía Tropical No. 20, Fraccionamiento Las Playas, C.P. 39390, Acapulco Guerrero, México.rfloresgarza@yahoo.com

${ }^{3}$ Universidad de Guadalajara, Departamento de Estudios para el Desarrollo Sustentable de Zonas Costeras, Av. Gómez Farías No. 82, C.P. 48980, San Patricio-Melaque Jalisco, México

${ }^{4}$ Facultad de Ciencias Biológicas, Universidad Autónoma de Nuevo León, Cd. Universitaria, San Nicolás de los Garza, Nuevo León, México

Resumen.- El objetivo de esta investigación fue la descripción de la taxocenosis de las subclases Prosobranchia y Pulmonata (clase Gastropoda), asociados a la zona intermareal rocosa de la Región Marina Prioritaria 32, Guerrero, México. Basada en la riqueza de especies, la composición de la comunidad a partir de la representación de las familias, se evaluó la abundancia, distribución geográfica de las especies, se estimó los estadísticos descriptivos de las tallas (en largo) de las poblaciones y la diversidad. El muestreo se llevó a cabo en 7 sitios, la unidad de muestreo fue de $1 \mathrm{~m}^{-2}$ y el área de muestreo de $10 \mathrm{~m}^{-2}$. Se identificaron 104 especies de prosobranquios y 4 de pulmonados. Las familias Columbellidae y Muricidae fueron las mejores representadas en riqueza de especies y la familia Muricidae en abundancia. Acanthais triangularis se consideró como la representativa de la zona, la especie Macrocypraea cervinetta mostró la mayor talla. Además, 23 especies presentaron amplia distribución, 27 distribución regular y 54 distribución restringida. Veintiocho especies estuvieron representadas por un único organismo, y 6 especies por 2 organismos, estas especies son consideradas raras. Las especies raras se encontraron en las estaciones cuya característica de exposición del oleaje es de bajo a medio. La riqueza de especies fue alta y corresponde a lo esperado en una zona tropical.
\end{abstract}

Palabras clave: Gastropoda, riqueza, distribución geográfica, tallas, Guerrero

\begin{abstract}
The aim of this research was the description of the taxocoenosis of Prosobranchia and Pulmonata subclasses (Gastropoda) associated with the rocky intertidal zone of the Marine Priority Region 32, Guerrero, Mexico. Based on known species richness, we examine the composition of the community based on the representation of families, assess abundance, the geographic distribution of species, estimate descriptive statistics of size, and estimate diversity. Sampling was conducted at 7 sites, the sampling unit was $1 \mathrm{~m}^{-2}$ and the sampling area was $10 \mathrm{~m}^{-2}$. One hundred and four species of subclass Prosobranchia and 4 species of subclass Pulmonata were identified. Columbellidae and Muricidae families showed higher species richness, meanwhile family Muricidae was the most abundant. Acanthais triangularis was the most representative species of the study area, and Macrocypraea cervinetta showed the greatest size. Twenty three species were found with broad distribution, 27 taxa with frequent distribution and 54 taxa with restricted distribution. Twenty eight species were represented by a single organism and 6 species with 2 organisms, these species were considered rare and were found in sites whose characteristic wave exposure is low to medium. Species richness was high and corresponds to that expected in the tropics.
\end{abstract}

Key words: Gastropoda, species richness, geographic distribution, size, Guerrero

\section{INTRODUCTION}

The state of Guerrero is considered one of the most biodiverse states of the Mexican Republic and the National Commission for the Conservation and Use of Biodiversity (initials in Spanish
CONABIO) determined the existence of 4 marine priority regions $(30,31,32$, and 33$)$ for the conservation and use of biodiversity in the state of Guerrero and reported that knowledge 
and understanding of biodiversity in these regions is limited (Arriaga et al. 1998).

The rocky intertidal zone of the Marine Priority Region 32 (MPR 32) is mainly located in the municipality of Acapulco. This rocky substratum is exposed to constant dives and rises, therefore its environmental conditions have vary greatly therefore allowing the development of many forms of life.

The molluscs of Gastropoda class, are part of the marine fauna that inhabits the MPR 32. These organisms are characterized by shells with various shapes and colors, some of them with an internal shell or without it at all. The main feature of the shell consists on its spiral shape. In the Gastropoda class not all organisms in their adulthood have a spiral shell, some present it before being adults and loose it in the process of growing into the mature stagestate (López \& Urcuyo 2009).

In Mexico, the Gastropoda class is very important for the ecosystem services it provides, besides many species of this class are used as food, most of them to prepare local gourmet dishes, other species are used in the industry for fashion, jewelry and handicrafts products. This class has contributed to the development and evolution of many of the cultures that formed this country.

In the American Pacific, has been conducted research that deal with the taxonomy and distribution of the Gastropoda class (Keen 1971, Brusca 1980, Skoglund 2002, Tucker \& Tenorio 2009, Tenorio et al. 2012). Meanwhile for the Mexican Pacific, in taxonomy, diversity, abundance, community composition, zonation, variation through time and geographical distribution of species (Holguín \& González 1989, Reguero \& GarcíaCubas 1989, Román et al. 1991, Holguín-Quiñones \& González-Pedraza 1994, Ríos-Jara et al. 1996, 2009; LandaJaime \& Arciniega-Flores 1998, Esqueda et al. 2000, Olabarría \& Vega 2000, Villarroel et al. 2000, González-Villareal 2005, Landa-Jaime et al. 2007, 2013; Ortíz-Arellano \& FloresCampaña 2008, Vega et al. 2008, Zamorano et al. 2008, Flores-Rodríguez et al. 2010, 2014). Particularly for the coasts of Guerrero there are reports where the Gastropoda class is analyzed as part of the set of classes studied (Salcedo et al. 1988, Flores 2004, Flores-Rodríguez et al. 2007, 2012). For the MPR 32 there are reports that have analyzed the molluscs at different sites (Villalpando 1986, Delgado 1989, García 1994, Flores-Rodríguez et al. 2003, Barba-Marino et al. 2010, Torreblanca 2010, Flores-Garza et al. 2010, 2011; Torreblanca et al. 2012, Torreblanca-Ramírez et al. 2012, 2014).

Concerning the Gastropoda class in the MPR 32, there is need for many investigations such as conducting inventory of species, record the composition of communities and populations, since this knowledge is essential to preserve and rationally use its biodiversity. The objective of this research was to analyze the composition of the Prosobranchia - Pulmonata taxocoenosis associated with the rocky intertidal zone of the MPR 32, based on known species richness, examine the composition of the community based on the representation of families, assess abundance, the geographic distribution of species, estimate descriptive statistics of size and the index of species diversity.

\section{MATERIALS AND METHODS}

In the marine ecoregion 17, is also called the Mexican Pacific Transitional region, besides the Guerrero coastal area in this ecoregion, the coasts of the states of Jalisco, Colima, Michoacán, Oaxaca and the southern tip of Baja California Sur are included. The physiography of this ecoregion is characterized by a narrow continental shelf with an amplitude of 10-15 km and a slope of less than 1 ${ }^{\circ} 30^{\prime}$, with a deep ocean trench and complex abyssal plains (Wilkinson et al. 2009).

The state of Guerrero has $470 \mathrm{~km}$ on costal length (Carranza-Edwards et al. 1986) and the MPR 32 is located between the Coyuca lagoon and Tres Palos lagoon (latitude $16^{\circ} 35^{\prime} 24^{\prime \prime} \mathrm{N}, 17^{\circ} 28^{\prime} 12^{\prime \prime} \mathrm{W}$ and longitude 99 $25^{\prime} 12^{\prime \prime} \mathrm{N}$, $100^{\circ} 33^{\prime}$ W) (Arriaga et al. 1998) (Fig. 1). Sampling was conducted at 7 sites at the rocky intertidal zone of this region (Fig. 1). Each location was geographically referenced and described according to the following criteria: length of the sampling area, type of substrate, types of rock, substrate stability and wave exposure. The descriptions of the collecting sites were based on Mottana et al. (1980) and maps (Acapulco E14-11, 1:50,000) produced by the National Institute of Statistics and Geography (Instituto Nacional de Estadística y Geografía. INEGI) and were complemented with field observations (Table 1).

These sites vary in type, substrate stability and wave exposure. Substrate types were classified as: 1) massif rocks: fixed structures such as walls, cliffs, terraces, etc.; 2) blocks: loose rock larger than $50 \mathrm{~cm}$ in diameter, made of rocks that are submerged and immovable or difficult to move about by the impact of waves, 3) boulders: loose rock with a size of less than $50 \mathrm{~cm}$ and greater than $8 \mathrm{~cm}$ in diameter, rocks that can be easily moved by the impact of waves, 4 ) gravel: loose rock no more than $8 \mathrm{~cm}$ in diameter, 5) artificial substrate: concrete blocks or construction waste.

The stability of the substrate was classified as: 1) high: when the substrate remains virtually unchanged by the impact of waves, 2) middle: when the impact of the waves does not change the configuration of the substrate but there is rock movement, 3 ) 


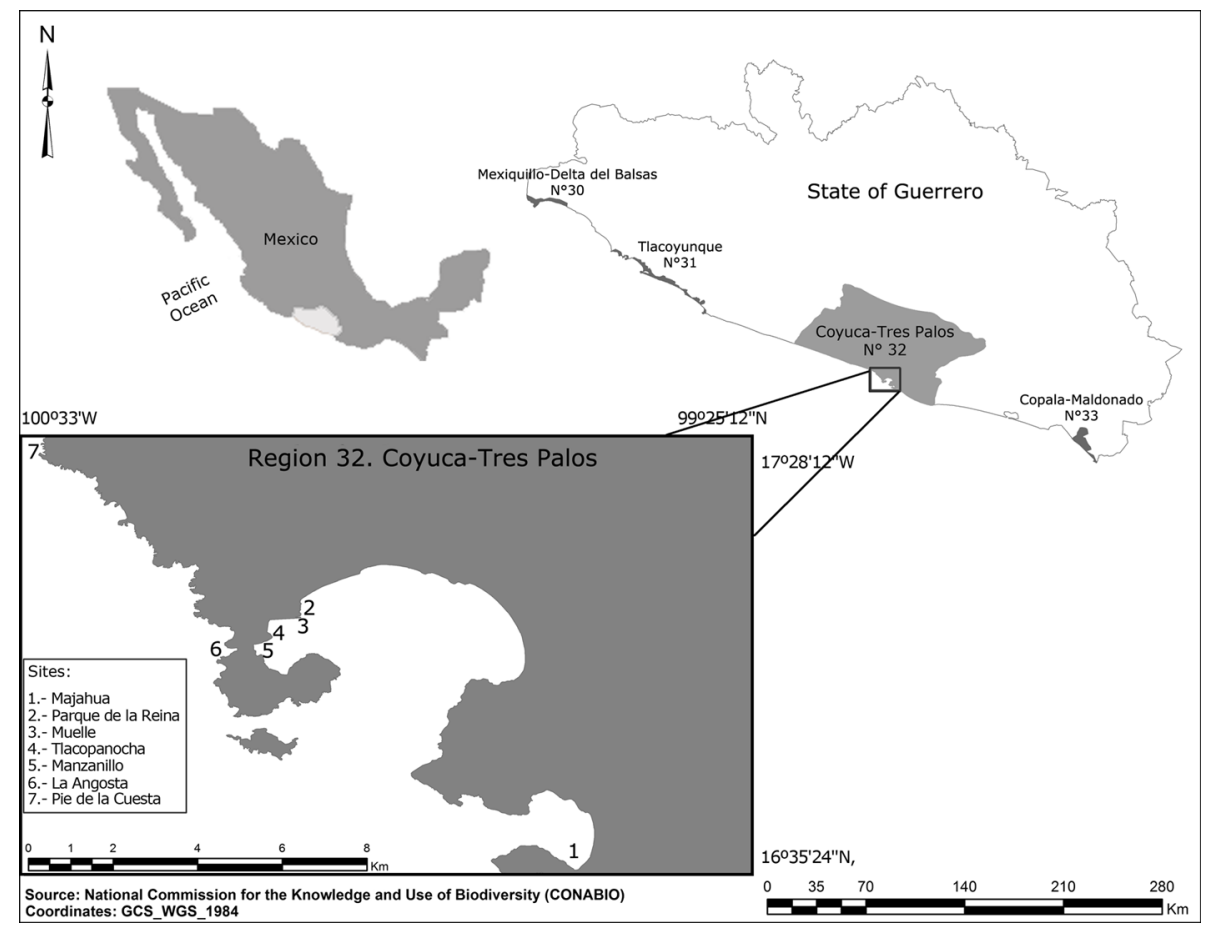

Figure 1. M arine Priority Regions in the state of Guerrero, Mexico and sampling sites / Regiones Marinas Prioritarias del Estado de Guerrero, México y sitios de muestreo

Table 1. Geographical location and the most relevant characteristics of the sampling sites / Localización geográfica y las características más relevantes de los sitios de muestreo

\begin{tabular}{|c|c|c|c|c|c|c|c|}
\hline \multirow[t]{2}{*}{ Site } & \multicolumn{2}{|c|}{ Coordinates } & \multirow{2}{*}{$\begin{array}{l}\text { Length } \\
\text { of the site } \\
\text { (m) }\end{array}$} & \multirow{2}{*}{$\begin{array}{l}\text { Type of } \\
\text { substrate }\end{array}$} & \multirow[t]{2}{*}{ Type of rock } & \multirow{2}{*}{$\begin{array}{l}\text { Substrate } \\
\text { stability }\end{array}$} & \multirow{2}{*}{$\begin{array}{c}\text { Wave } \\
\text { exposure }\end{array}$} \\
\hline & Latitude N & LongitudeW & & & & & \\
\hline Majahua & $16^{\circ} 47^{\prime} 39.46^{\prime \prime}$ & $99^{\circ} 50^{\prime} 28.94^{\prime \prime}$ & 600 & $\begin{array}{l}\text { Blocks and } \\
\text { Boulders }\end{array}$ & Metamorphic & High & Low \\
\hline $\begin{array}{l}\text { Parque de la } \\
\text { Reina }\end{array}$ & $16^{\circ} 51^{\prime} 01.21^{\prime \prime}$ & $99^{\circ} 54^{\prime} 02.04^{\prime \prime}$ & 66.74 & $\begin{array}{l}\text { Boulders and } \\
\text { Gravel }\end{array}$ & $\begin{array}{l}\text { Artificial substrate and } \\
\text { igneous rocks }\end{array}$ & Low & Middle \\
\hline Muelle & $16^{\circ} 50^{\prime} 56.86^{\prime \prime}$ & $99^{\circ} 54^{\prime} 02.39^{\prime \prime}$ & 60 & $\begin{array}{l}\text { Blocks and } \\
\text { Boulders }\end{array}$ & $\begin{array}{l}\text { Metamorphic and } \\
\text { artificial substrate }\end{array}$ & Meddle & Middle \\
\hline Tlacopanocha & $16^{\circ} 50^{\prime} 41.53^{\prime \prime}$ & $99^{\circ} 54^{\prime} 25.02^{\prime \prime}$ & 200 & $\begin{array}{l}\text { Blocks and } \\
\text { Gravel }\end{array}$ & $\begin{array}{l}\text { Artificial substrate and } \\
\text { igneous rocks }\end{array}$ & Meddle & Middle \\
\hline Manzanillo & $16^{\circ} 50^{\prime} 27.90^{\prime \prime}$ & $99^{\circ} 54^{\prime} 38.14^{\prime \prime}$ & 22.87 & $\begin{array}{c}\text { Boulders and } \\
\text { Gravel }\end{array}$ & $\begin{array}{l}\text { Metamorphic and } \\
\text { artificial substrate }\end{array}$ & Low & Low \\
\hline La Angosta & $16^{\circ} 60^{\prime} 29.86^{\prime \prime}$ & $99^{\circ} 54^{\prime} 55.70^{\prime \prime}$ & 48.14 & $\begin{array}{l}\text { Massif rocks } \\
\text { and Boulders }\end{array}$ & Metamorphic & High & High \\
\hline Pie de la Cuesta & $16^{\circ} 52^{\prime} 25.64^{\prime \prime}$ & $99^{\circ} 56^{\prime} 34.64^{\prime \prime}$ & 66 & $\begin{array}{l}\text { Massif rocks } \\
\text { and blocks }\end{array}$ & Metamorphic & High & High \\
\hline
\end{tabular}


low: when the site configuration is changed by the impact of waves, most of the rocks are moved. The wave exposure was classified as: 1) high: when the wave hits the substrate in a free manner, usually the sites that have this type of waves are outside of the protection of barriers such as bays or hooks, 2) middle: when the impact of the wave on the substrate is hindered by barriers or smoothing, as can occur at sites that are in front of the entrance of bays or at a distance from a wall of hooks, it also happens in places where subtidal rocky substrate emerges or is shallow and reduces the direct impact of the waves to the substrate, 3) low: when the wave does not directly hit the substrate, since the sites are protected by different types of barriers (Flores-Garza et al. 2012).

Three samplings were conducted per site between 2010 and 2012. Each sampling was performed during the hours of low tide and new moon days, the sampling area was $10 \mathrm{~m}^{-2}$ and sampling unit was $1 \mathrm{~m}^{-2}$. Sampling was systematic. The starting point of the sample was randomly selected in the rocky intertidal zone and the sampling unit was delimited using a $1 \mathrm{~m}^{-2}$ frame made of PVC pipe. A rope $30 \mathrm{~m}$ in length, was placed next to the PVC frame, which extended parallel to the coast over the rocky intertidal zone, in order to define the transect on which sampling would be carried out.

Gastropods found alive within the sampling unit, were collected and placed into a plastic container filled with seawater and protected from direct sunlight. The following sampling unit was placed along the transect $2 \mathrm{~m}$ from the first sampling unit. This procedure was repeated to complete $10 \mathrm{~m}^{-2}$. The specimens were placed in bottles containing $96 \%$ ethyl alcohol and taken to the laboratory to be identified and quantified.

The identification of specimens and the update of the nomenclature, required confirmation in the laboratory, using literature such as Keen (1971), Skoglund (2002) and WoRMS $(2015)^{1}$.

The collected specimens were measured in length and width (mm). Species richness was assessed based on the number of species found in the samples. The community composition was analyzed using the representation of families, which was evaluated based on species richness and abundance of organisms per family and expressed as percentage. Relative abundance was measured as the percentage of each species in relation to the total number of specimens. The geographical distribution was evaluated based on the frequency of occurrence of species per site as follows: broad (frequency of 6 to 7 sites), frequent (frequency of 3 to 5 sites) and restricted (frequency of 1 to 2 sites). The composition analysis of the Gastropoda sizes was considered by measuring the length and obtaining the descriptive statistical values such as maximum, minimum, mean and standard deviation for each of the species. The diversity was measured using the Shannon-Wiener index $\left(\mathrm{H}^{\prime}\right)$ and Pielou index $\left(J^{\prime}\right)$.

\section{Results}

We examined 12,881 specimens collected in the rocky intertidal zone of the MPR 32, from which 11,694 specimens belonged to the subclass Prosobranchia and 1,187 specimens belonged to the subclass Pulmonata. In total 29 families, 63 genera and 108 species were identified. The subclass Prosobranchia was composed of 28 families, 61 genera and 104 species and subclass Pulmonata by 1 family, 2 genera and 4 species (Table 2) (Figs. 2 and 3).

In subclass Prosobranchia, the Columbellidae family was the best represented in species richness (10 genera and 19 species), followed by families Muricidae (10 genera and 13 species), Fissurellidae (3 genera and 13 species), Calyptraeidae ( 3 genera and 9 species) and Lottiidae ( 2 genera and 6 species). Families found represented by a single species were: Planaxidae, Epitoniacea, Naticidae, Triviidae, Ovulidae, Cymantiidae, Melongenidae, Nassariidae, Olividae and Cancellariidae (Table 2). The Siphonariidae family, subclass Pulmonata was represented by 4 species.

The site with the highest species richness in both subclasses analyzed was Majahua, in which 74 species (70 prosobranchs and 4 pulmonates) were identified, in second place was Tlacopanocha (63 prosobranchs and 2 pulmonates), and followed by Manzanillo ( 55 prosobranchs and 2 pulmonates). The lower species richness was found on La Angosta (35 prosobranchs and 2 pulmonates) and Pie de la Cuesta (30 prosobranchs and 2 pulmonates) (Table 3 ).

In the MPR 32 of the subclass Prosobranchia, the family with the best representation in relative abundance was Muricidae (29.04\%), followed by Columbellidae (26.10\%), Lottiidae $(9.02 \%)$, and Trochidae $(8.89 \%)$. The family Siphonariidae included in the Subclass Pulmonata also found within families had higher relative abundance (9.21\%). 12 families had a value greater than $1 \%$ of relative abundance. Together these 12 families accounted for $97.09 \%$ of the total abundance found in the MRP 32. The families lowest value recorded in relative abundance were Triviidae $(0.023 \%)$, Turridae $(0.046 \%)$ and Cerithiidae (0.147\%).

The Columbellidae family in 6 sites was found as 1 of the 2 most abundant families, The Muricidae family was found in 5 sites and Siphonariidae, Trochidae and Fissurellidae families on 1 site (Table 3). 
Table 2. Families and species, relative abundance, geographical distribution and descriptive statistics of sizes in length of species Prosobranchia and Pulmonata subclasses, associated with the rocky intertidal zone of The MPR 32, Guerrero, Mexico / Familias y especies, abundancia relativa, distribución geográfica y estadísticos descriptivos talla en largo de las especies Prosobranchia y Pulmonata subclases, asociados a la zona intermareal rocosa de la RMP 32, Guerrero, México

\begin{tabular}{|c|c|c|c|c|c|c|c|c|c|c|c|c|c|c|}
\hline \multirow{2}{*}{ Families / Species } & \multirow[b]{2}{*}{$\mathrm{N}$} & \multirow[b]{2}{*}{1} & \multicolumn{5}{|c|}{ Sampling sites } & \multirow{2}{*}{\multicolumn{2}{|c|}{$\begin{array}{c}\mathrm{RAB} \\
\mathrm{Sp}\end{array}$}} & \multirow{2}{*}{ Gd } & \multicolumn{4}{|c|}{ Length (mm) } \\
\hline & & & 2 & 3 & 4 & 5 & 6 & & & & Min & $\operatorname{Max}$ & Ave & $\mathrm{Sd}$ \\
\hline \multicolumn{15}{|l|}{ Subclass Prosobranchia } \\
\hline \multicolumn{15}{|l|}{ FISSURELLIDAE } \\
\hline Octomarginula natlandi (Durham, 1950) & 1 & 1 & & & & & & & 0.01 & $\mathrm{R}$ & 15.2 & 15.1 & 15.1 & 15.1 \\
\hline Diodora digueti (Mabille, 1895) & 1 & 1 & & & & & & & 0.01 & $\mathrm{R}$ & 10.5 & 10.4 & 10.4 & 10.4 \\
\hline Diodora inaequalis (G.B. Sowerby I, 1835) & 35 & 6 & 4 & & 3 & 20 & 2 & & 0.27 & $\mathrm{~F}$ & 8.8 & 25.5 & 17.2 & 4.2 \\
\hline Diodora saturnalis (Carpenter, 1864) & 10 & & & & 2 & 8 & & & 0.08 & $\mathrm{R}$ & 6.7 & 23.9 & 15.8 & 5.8 \\
\hline Fissurella asperella G.B. Sowerby I, 1835 & 1 & & 1 & & & & & & 0.01 & $\mathrm{R}$ & 16.9 & 16.9 & 16.9 & \\
\hline Fissurella decemcostata McLean, 1970 & 5 & 4 & & 1 & & & & & 0.04 & $\mathrm{R}$ & 15.0 & 20.6 & 17.0 & 2.1 \\
\hline Fissurella deroyae McLean, 1970 & 2 & 1 & 1 & & & & & & 0.02 & $\mathrm{R}$ & 11.2 & 12.8 & 12.0 & 1.1 \\
\hline Fissurella gemmata Menke, 1847 & 154 & & & & & & & 154 & 1.20 & $\mathrm{R}$ & 5.8 & 27.4 & 21.1 & 3.3 \\
\hline Fissurella macrotrema G. B. Sowerby I, 1835 & 1 & & 1 & & & & & & 0.01 & $\mathrm{R}$ & 9.8 & 9.8 & 9.8 & \\
\hline Fissurella microtrema G. B. Sowerby, 1835 & 5 & 1 & & & & 4 & & & 0.04 & $\mathrm{R}$ & 6.7 & 32.4 & 20.4 & 9.5 \\
\hline Fissurella nigrocincta Carpenter, 1856 & 227 & & & & & 2 & & 225 & 1.76 & $\mathrm{R}$ & 6.5 & 27.8 & 18.8 & 4.3 \\
\hline Fissurella obscura G. B. Sowerby I, 1834 & 1 & & 1 & & & & & & 0.01 & $\mathrm{R}$ & 22.0 & 22.03 & 22.0 & \\
\hline Fissurella rubropicta Plisbry, 1890 & 1 & & 1 & & & & & & 0.01 & $\mathrm{R}$ & 8.5 & 8.5 & 8.5 & \\
\hline \multicolumn{15}{|l|}{ LOTTIIDAE } \\
\hline Lottia acutapex (S. S. Berry, 1960) & 29 & & & & & & 28 & 1 & 0.23 & $\mathrm{R}$ & 2.41 & 17.02 & 5.14 & 2.71 \\
\hline Lottia mitella (Menke, 1847) & 98 & & & & 3 & 4 & 49 & 42 & 0.76 & $\mathrm{~F}$ & 3.45 & 17.95 & 8.45 & 3.69 \\
\hline Lottia mesoleuca (Menke, 1851) & 146 & 76 & 11 & 11 & 5 & 1 & 2 & 40 & 1.13 & B & 9.36 & 30.51 & 17.83 & 4.36 \\
\hline Lottia pediculus (Philippi, 1846) & 289 & 2 & 2 & 38 & 54 & 1 & 192 & & 2.24 & B & 4.11 & 26.08 & 12.35 & 3.7 \\
\hline Lottia fascicularis (Menke, 1851) & 599 & 34 & 12 & 276 & 98 & 21 & 108 & 50 & 4.65 & B & 4.62 & 31.85 & 15.25 & 5.75 \\
\hline Patelloida semirubida (Dall, 1914) & 2 & 1 & & & 1 & & & & 0.02 & $\mathrm{R}$ & 5.82 & 11.59 & 8.7 & 4.08 \\
\hline \multicolumn{15}{|l|}{ TROCHIDAE } \\
\hline Calliostoma aequisculptum Carpenter, 1865 & 2 & 2 & & & & & & & 0.02 & $\mathrm{R}$ & 16.9 & 16.85 & 16.85 & \\
\hline Tegula globulus (Carpenter, 1857) & 1128 & 64 & 199 & 843 & & 18 & & 4 & 8.76 & $\mathrm{~F}$ & 5,47 & 9.72 & 7.21 & 1.09 \\
\hline Tegula panamensis (Philippi, 1849) & 4 & 2 & & 2 & & & & & 0.03 & $\mathrm{R}$ & 6.34 & 11.13 & 8.35 & 2.32 \\
\hline Monilea patricia (Philippi, 1851) & 12 & 10 & 1 & & & & 1 & & 0.09 & $\mathrm{~F}$ & 4,21 & 10.33 & 7.05 & 1.98 \\
\hline TURBINIDAE & & & & & & & & & & & & & & \\
\hline Arene hindsiana Pilsbry \& Lowe, 1932 & 3 & 2 & 1 & & & & & & 0.02 & $\mathrm{R}$ & 5.47 & 6.67 & 6.07 & 0.84 \\
\hline Uvanilla unguis (W. Wood, 1828) & 21 & 11 & 8 & 2 & & & & & 0.16 & $\mathrm{~F}$ & 8.76 & 41.22 & 17.93 & 8.3 \\
\hline NERITIDAE & & & & & & & & & & & & & & \\
\hline Nerita scabricosta Lamarck, 1822 & 26 & 5 & 9 & 5 & & 3 & & 4 & 0.20 & $\mathrm{~F}$ & 9.28 & 17.64 & 12.13 & 2.29 \\
\hline Nerita funiculata Menke, 1851 & 105 & 50 & 3 & 1 & & 50 & & 1 & 0.82 & $\mathrm{~F}$ & 3.64 & 15.18 & 8.24 & 2.46 \\
\hline Eulithidium perforatum (Philippi, 1848) & 1 & & & & 1 & & & & 0.01 & $\mathrm{R}$ & 3.12 & 3.12 & 3.12 & \\
\hline LITTORINIDAE & & & & & & & & & & & & & & \\
\hline Echinolittorina aspera (Philippi 1846) & 205 & 65 & 70 & 58 & & 9 & & 3 & 1.59 & $\mathrm{~F}$ & 2.54 & 13.43 & 6.61 & 3.09 \\
\hline Echinolittorina peruviana (Lamarck, 1828) & 3 & & & 3 & & & & & 0.02 & $\mathrm{R}$ & 6,1 & 6.41 & 6.29 & 0.16 \\
\hline Echinolittorina modesta (Philippi, 1846) & 113 & 36 & 15 & & 8 & & 8 & 46 & 0.88 & $\mathrm{~F}$ & 1.89 & 15.4 & 7.96 & 3.45 \\
\hline RISSOIDAE & & & & & & & & & & & & & & \\
\hline Rissoina stricta (Menke, 1850) & 66 & 1 & 2 & 52 & 9 & 1 & 1 & & 0.51 & B & 4.63 & 8.09 & 6.59 & 0.66 \\
\hline CERITHIIDAE & & & & & & & & & & & & & & \\
\hline Cerithium menkei Carpenter, 1857 & 4 & 1 & & & & 3 & & & 0.03 & $\mathrm{R}$ & 6.55 & 8.22 & 7.31 & 0.84 \\
\hline Cerithium gemmatum Hinds, 1844 & 3 & 1 & & 2 & & & & & 0.02 & $\mathrm{~F}$ & 6.98 & 9.93 & 8.45 & 2.08 \\
\hline Seila assimilata (C. B. Adams, 1852) & 6 & 1 & & 2 & 3 & & & & 0.05 & $\mathrm{R}$ & 4.28 & 7.51 & 5.27 & 1.13 \\
\hline PLANAXIDAE & & & & & & & & & & & & & & \\
\hline Planaxis obsoletus Menke, 1851 & 154 & 59 & 1 & 19 & 11 & & 5 & 59 & 1.20 & B & 4.77 & 16.42 & 12.12 & 2.39 \\
\hline EPITONIIDAE & & & & & & & & & & & & & & \\
\hline Epitonium cookeanum Dall, 1917 & 2 & & & 2 & & & & & 0.02 & $\mathrm{R}$ & 7.28 & 8.07 & 7.67 & 0.55 \\
\hline HIPPONICIDAE & & & & & & & & & & & & & & \\
\hline Hipponix panamensis C. B. Adams, 1852 & 9 & 2 & 1 & & & 6 & & & 0.07 & $\mathrm{~F}$ & 6.93 & 28.67 & 12.74 & 6.31 \\
\hline Hipponix delicatus Dall, 1908 & 19 & 14 & 1 & & 3 & 1 & & & 0.15 & $\mathrm{~F}$ & 6.47 & 14.09 & 10.03 & 2.06 \\
\hline Pilosabia trigona (Gmelin, 1791) & 1 & & & & & 1 & & & 0.01 & $\mathrm{R}$ & 16.12 & 16.12 & 16.12 & \\
\hline CALYPTRAEIDAE & & & & & & & & & & & & & & \\
\hline Bostrycapulus acueleatus (Gmelin, 1791) & 122 & 30 & 4 & & 2 & 86 & & & 0.95 & $\mathrm{~F}$ & 6.95 & 27.99 & 18.97 & 4.49 \\
\hline Crepidula excavata (Broderip 1834) & 31 & 2 & & & & 29 & & & 0.24 & $\mathrm{R}$ & 8.4 & 30.42 & 19.52 & 4.5 \\
\hline Crepidula incurva (Broderip, 1834) & 32 & 19 & 3 & 2 & & 8 & & & 0.25 & $\mathrm{~F}$ & 4.2 & 11.99 & 8.11 & 2.3 \\
\hline Crepidula onyx G. B. Sowerby, 1824 & 5 & & 5 & & & & & & 0.04 & $\mathrm{R}$ & 13.6 & 13.64 & 13.64 & \\
\hline Crepidula striolata Menke, 1851 & 9 & 2 & 1 & & & 6 & & & 0.07 & $\mathrm{~F}$ & 9.8 & 36.04 & 22.02 & 7.7 \\
\hline Crucibullum subactum Berry, 1963 & 31 & 2 & 2 & 2 & 2 & 10 & 10 & 3 & 0.24 & B & 3.08 & 26.12 & 10.57 & 5.2 \\
\hline Crucibulum umbrella (Deshayes, 1830) & 230 & 142 & 53 & 13 & 8 & 10 & 3 & 1 & 1.79 & B & 8.39 & 65.85 & 38.29 & 10.99 \\
\hline
\end{tabular}


Table 2. Continued / Continuación

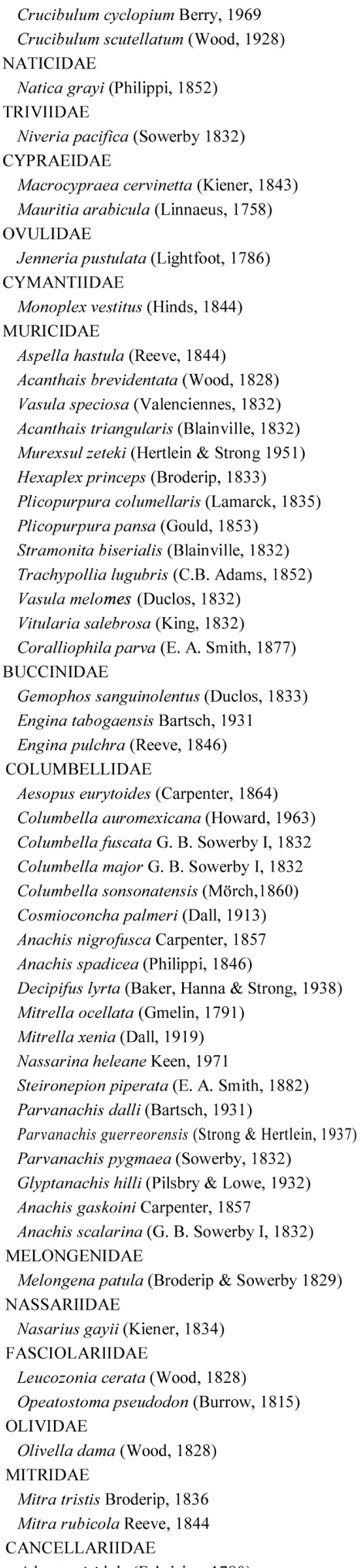

\begin{tabular}{|c|c|c|c|c|c|c|c|c|c|c|c|c|c|}
\hline 14 & & 3 & & & 11 & & & 0.11 & $\mathrm{R}$ & 26.2 & 59.25 & 44.58 & 10.67 \\
\hline 32 & 17 & 2 & 1 & 2 & 4 & 3 & 3 & 0.25 & B & 11.2 & 43.06 & 23.38 & 10.11 \\
\hline 1 & 1 & & & & & & & 0.01 & $\mathrm{R}$ & 2.32 & 2.32 & 2.32 & \\
\hline 3 & 1 & & & & 2 & & & 0.02 & $\mathrm{R}$ & 8.48 & 10.15 & 9.31 & 1.18 \\
\hline 20 & 2 & 7 & 7 & 1 & 2 & 1 & & 0.16 & B & 7.97 & 84.48 & 37.82 & 24.12 \\
\hline 44 & 7 & 14 & 8 & 4 & 11 & & & 0.34 & $\mathrm{~F}$ & 17.8 & 31.27 & 23.47 & 3.47 \\
\hline 24 & 5 & 3 & 1 & & 15 & & & 0.19 & $\mathrm{~F}$ & 10.1 & 18.91 & 14.48 & 2.66 \\
\hline 1 & 1 & & & & & & & 0.01 & $\mathrm{R}$ & 49.6 & 49.63 & 49.63 & \\
\hline 1 & & 1 & & & & & & 0.01 & $\mathrm{R}$ & & & & \\
\hline 4 & 1 & 1 & & & & 2 & & 0.03 & $\mathrm{~F}$ & 19.12 & 25.28 & 22.76 & 3.04 \\
\hline 228 & 21 & 26 & 54 & 23 & 18 & 64 & 22 & 1.77 & B & 5.5 & 28.88 & 16.55 & 4.83 \\
\hline 1642 & 149 & 41 & 724 & 41 & 36 & 452 & 199 & 12.75 & B & 3.11 & 17.09 & 10 & 2.34 \\
\hline 45 & 6 & 2 & 6 & 1 & 30 & & & 0.35 & $\mathrm{~F}$ & 7.73 & 48.96 & 17.05 & 5.93 \\
\hline 1 & & & & & 1 & & & 0.01 & $\mathrm{R}$ & 49.1 & 49.1 & 49.1 & \\
\hline 7 & & & & 1 & 1 & 2 & 3 & 0.05 & F & 17.04 & 26.52 & 21.95 & 3.52 \\
\hline 433 & 3 & & 4 & 36 & 3 & 266 & 121 & 3.36 & B & 10.12 & 34.53 & 18.84 & 4.37 \\
\hline 774 & 85 & 19 & 376 & 57 & 84 & 76 & 77 & 6.01 & B & 3.88 & 50.56 & 12.61 & 6.01 \\
\hline 601 & 25 & 5 & 213 & 23 & 28 & 304 & 3 & 4.67 & B & 2.73 & 25.88 & 13.81 & 4.15 \\
\hline 1 & & & 1 & & & & & 0.01 & $\mathrm{R}$ & 39.15 & 39.15 & 39.15 & \\
\hline 3 & 3 & & & & & & & 0.02 & $\mathrm{R}$ & 15.65 & 19.99 & 18.53 & 2.49 \\
\hline 1 & & & 1 & & & & & 0.01 & $\mathrm{R}$ & 9.05 & 9.05 & 9.05 & \\
\hline 59 & 11 & 6 & 7 & 4 & 17 & 14 & & 0.46 & $\mathrm{~F}$ & 15.3 & 27.58 & 20.89 & 2.7 \\
\hline 131 & 67 & 7 & 3 & 2 & 46 & 6 & & 1.02 & $\mathrm{~F}$ & 4.43 & 14.71 & 10.45 & 2.09 \\
\hline 1 & & & & 1 & & & & 0.01 & $\mathrm{R}$ & 6.45 & 6.45 & 6.45 & \\
\hline 1 & & 1 & & & & & & 0.01 & $\mathrm{R}$ & & & & \\
\hline 5 & 1 & 1 & 2 & 1 & & & & 0.04 & $\mathrm{~F}$ & 5.82 & 14.92 & 10.85 & 4.62 \\
\hline 693 & 154 & 25 & 334 & 72 & 76 & 29 & 3 & 5.38 & B & 3.87 & 26.98 & 14.84 & 4.05 \\
\hline 72 & 6 & 13 & 1 & 2 & 32 & 18 & & 0.56 & B & 16.2 & 30.3 & 22.39 & 2.51 \\
\hline 38 & 7 & 4 & 3 & 1 & 5 & 18 & & 0.30 & B & 6.36 & 17.73 & 10.32 & 3.06 \\
\hline 20 & 7 & 3 & 2 & 7 & & 1 & & 0.16 & $\mathrm{~F}$ & 6,71 & 10.99 & 8.75 & 1.11 \\
\hline 643 & 58 & 36 & 381 & 80 & 72 & 13 & 3 & 4.99 & B & 2.68 & 17.67 & 7.13 & 1.04 \\
\hline 1 & & 1 & & & & & & 0.01 & $\mathrm{R}$ & 5.25 & 5.25 & 5.25 & \\
\hline 6 & 1 & 1 & 4 & & & & & 0.05 & $\mathrm{R}$ & 7.34 & 8.77 & 7.95 & 0.49 \\
\hline 1001 & 32 & 9 & 273 & 47 & 81 & 432 & 127 & 7.77 & B & 4.92 & 13.06 & 10.35 & 1.24 \\
\hline 499 & 13 & 3 & 5 & 8 & 11 & 416 & 43 & 3.87 & B & 4.14 & 14.86 & 8.4 & 1.61 \\
\hline 1 & & 1 & & & & & & 0.01 & $\mathrm{R}$ & & & & \\
\hline 1 & & 1 & & & & & & 0.01 & $\mathrm{R}$ & 4.57 & 4.57 & 4.57 & \\
\hline 368 & 74 & 2 & 3 & 43 & 242 & 2 & 2 & 2.86 & B & 3.18 & 7.35 & 5.57 & 0.6 \\
\hline 8 & 1 & & & & 7 & & & 0.06 & $\mathrm{R}$ & 9.74 & 11.93 & 10.99 & 0.67 \\
\hline 2 & 1 & & 1 & & & & & 0.02 & $\mathrm{R}$ & 4.62 & 4.62 & 4.62 & \\
\hline 1 & & 1 & & & & & & 0.01 & $\mathrm{R}$ & 7.18 & 7.18 & 7.18 & \\
\hline 1 & & & 1 & & & & & 0.01 & $\mathrm{R}$ & 7.32 & 7.32 & 7.32 & \\
\hline 1 & & & & & 1 & & & 0.01 & $\mathrm{R}$ & 6.18 & 6.18 & 6.18 & \\
\hline 10 & & 4 & & & 4 & 2 & & 0.08 & $\mathrm{~F}$ & 25.86 & 30.11 & 28.18 & 1.7 \\
\hline 1 & & & & & 1 & & & 0.01 & $\mathrm{R}$ & 6.32 & 6.32 & 6.32 & \\
\hline 88 & 15 & 2 & 8 & 4 & 30 & 22 & 7 & 0.68 & B & 7.9 & 69.13 & 23.78 & 10.66 \\
\hline 79 & 1 & 4 & 19 & 3 & 4 & 48 & & 0.61 & B & 12.33 & 45.57 & 23.21 & 5.55 \\
\hline 1 & & & 1 & & & & & 0.01 & $\mathrm{R}$ & 7.37 & 7.37 & 7.37 & \\
\hline 45 & 7 & 2 & 4 & 31 & 1 & & & 0.35 & F & 3.51 & 16.2 & 10.03 & 2.77 \\
\hline 13 & 4 & & & & 9 & & & 0.10 & $\mathrm{R}$ & 8.27 & 27.18 & 15.08 & 6.05 \\
\hline 2 & 1 & & & 1 & & & & 0.02 & $\mathrm{R}$ & 4.85 & 11.41 & 8.13 & 4.63 \\
\hline
\end{tabular}

Torreblanca-Ramírez et al.

Composition of Prosobranchia-Pulmonata, Guerrero Mexico 


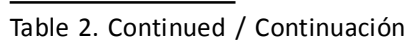

\begin{tabular}{|c|c|c|c|c|c|c|c|c|c|c|c|c|c|c|}
\hline \multicolumn{15}{|l|}{ CONIDAE } \\
\hline Conus brunneus Wood, 1828 & 1 & 1 & & & & & & & 0.01 & & & & & \\
\hline Conus purpurascens G. B. Sowerby I, 1833 & 1 & 1 & & & & & & & 0.01 & $\mathrm{R}$ & 40.11 & 40.11 & 40.11 & \\
\hline Conus gladiator Broderip, 1833 & 16 & 6 & 3 & & & 3 & 4 & & 0.12 & $\mathrm{~F}$ & 8.16 & 25.96 & 15.84 & 6.56 \\
\hline Conus nux Broderip, 1833 & 38 & 5 & 4 & & 5 & 24 & & & 0.30 & $\mathrm{~F}$ & 9.51 & 24.03 & 16.58 & 4.09 \\
\hline Conus princeps Linnaeus, 1758 & 1 & & & & 1 & & & & 0.01 & $\mathrm{R}$ & 31.6 & 31.6 & 31.6 & \\
\hline \multicolumn{15}{|l|}{ TURRIDAE } \\
\hline Pilsbryspira garciacubasi Shasky, 1971 & 5 & 4 & & 1 & & & & & 0.04 & $\mathrm{R}$ & 12.7 & 14.38 & 13.52 & 0.78 \\
\hline Crassispira cerithoidea (Carpenter, 1857) & 1 & 1 & & & & & & & 0.01 & $\mathrm{R}$ & 9.26 & 9.26 & 9.26 & \\
\hline \multicolumn{15}{|l|}{ Subclass Pulmonata } \\
\hline \multicolumn{15}{|l|}{ SIPHONARIIDAE } \\
\hline Siphonaria gigas G. B. Sowerby I, 1825 & 15 & 5 & & 8 & & & 2 & & 0.12 & F & 8.62 & 19.37 & 13.09 & 3.61 \\
\hline Siphonaria maura Sowerby I, 1835 & 784 & 564 & 4 & 64 & 35 & 81 & 14 & 22 & 6.09 & B & 3.62 & 19.52 & 9.67 & 2.69 \\
\hline Siphonaria palmata Carpenter, 1857 & 387 & 302 & 5 & 21 & 24 & 34 & & 1 & 3.00 & B & 3.78 & 16.74 & 8.71 & 2.09 \\
\hline Williamia peltoides (Carpenter, 1864) & 1 & 1 & & & & & & & 0.01 & $\mathrm{R}$ & 5.51 & 5.51 & 5.51 & \\
\hline
\end{tabular}

$\mathrm{N}=$ Number of organisms analyzed, $1=$ Majahua, $2=$ Tlacopanocha, $3=$ Parque de la Reina, $4=$ Muelle, $5=$ Manzanillo, $6=$ La Angosta y $7=$ Pie de la Cuesta, RAB $\mathrm{sp}=$ relative abundance of each species, $\mathrm{Gd}=$ Geographical distribution $(\mathrm{B}=\mathrm{Broad}, \mathrm{F}=$ Frecuent, $\mathrm{R}=$ Restricted $)$, Min= Minimum, Max= Maximum, Avg $=\mathrm{Average}$ $\mathrm{Sd}=$ Standard deviation

Table 3. Relative abundance and distribution of the families of Gastropods (Subclasses Prosobranchia and Pulmonata) associated with the rocky intertidal zone of the MPR 32, Guerrero, Mexico / Abundancia relativa y distribución de las familias de gasterópodos (Subclases Prosobranchia y Pulmonata) asociada a la zona intermareal rocoso de la RMP 32, Guerrero, México

\begin{tabular}{|c|c|c|c|c|c|c|c|c|}
\hline \multirow{2}{*}{ Families } & \multirow{2}{*}{ All sites } & \multicolumn{7}{|c|}{ Sampling sites } \\
\hline & & 1 & 2 & 3 & 4 & 5 & 6 & 7 \\
\hline FISSURELLIDAE & 3.44 & 0.04 & 1.32 & 0.25 & 0.64 & 2.56 & 0.07 & 29.86 \\
\hline LOTTIIDAE & 9.02 & 4.85 & 3.67 & 8.36 & 20.80 & 2.03 & 14.46 & 10.48 \\
\hline TROCHIDAE & 8.89 & 3.35 & 29.41 & 21.74 & & 1.35 & 0.03 & 0.31 \\
\hline TURBINIDAE & 0.18 & 0.55 & 1.32 & 0.05 & & & & \\
\hline NERITIDAE & 1.02 & 2.36 & 1.76 & 0.15 & 0.12 & 2.03 & & 0.39 \\
\hline LITTORINIDAE & 2.49 & 4.34 & 12.5 & 1.56 & 1.03 & 0.67 & 0.30 & 3.86 \\
\hline RISSOIDAE & 0.51 & 0.04 & 0.29 & 1.33 & 1.62 & 0.07 & 0.03 & \\
\hline CERITHIIDAE & 0.14 & 0.08 & & 0.36 & 0.38 & & & 4.64 \\
\hline PLANAXIDAE & 1.19 & 2.53 & 0.14 & 0.49 & 1.42 & & 0.19 & \\
\hline EPITONIIDAE & 0.01 & & & 0.05 & 0.38 & & & \\
\hline HIPPONICIDAE & 0.22 & 0.68 & 0.29 & & & 0.60 & & \\
\hline CALYPTRAEIDAE & 3.92 & 9.20 & 10.73 & 0.46 & 1.80 & 12.36 & 0.61 & 0.55 \\
\hline NATICIDAE & 0.007 & 0.04 & & & & & & \\
\hline TRIVIIDAE & 0.02 & 0.04 & & & & 0.15 & & \\
\hline CYPRAEIDAE & 0.49 & 0.38 & 3.08 & 0.38 & 0.64 & 0.98 & 0.03 & \\
\hline OVULIDAE & 0.18 & 0.21 & 0.44 & 0.02 & & 1.13 & & \\
\hline CYMANTIIDAE & 0.007 & 0.04 & & & & & & \\
\hline MURICIDAE & 29.04 & 12.59 & 13.97 & 35.48 & 23.51 & 15.15 & 44.50 & 33.49 \\
\hline BUCCINIDAE & 1.48 & 3.35 & 1.91 & 0.25 & 0.90 & 4.75 & 0.76 & \\
\hline COLUMBELLIDAE & 26.1 & 15.26 & 15 & 25.99 & 33.72 & 39.74 & 35.45 & 14.02 \\
\hline MELONGENIDAE & 0.07 & & 0.58 & & & 0.30 & 0.07 & \\
\hline NASSARIIDAE & 0.007 & & & & & 0.07 & & \\
\hline FASCIOLARIIDAE & 1.29 & 0.68 & 0.88 & 0.69 & 0.90 & 2.56 & 2.67 & 0.55 \\
\hline OLIVIDAE & 0.007 & & & 0.02 & & & & \\
\hline MITRIDAE & 0.45 & 0.47 & 0.58 & 0.10 & 4 & 0.75 & & \\
\hline CANCELLARIIDAE & 0.01 & 0.04 & & & 0.12 & & & \\
\hline CONIDAE & 0.43 & 0.51 & 1.02 & & 0.77 & 2.03 & 0.15 & \\
\hline TURRIDAE & 0.04 & 0.21 & & 0.25 & & & & \\
\hline SIPHONARIIDAE & 9.21 & 37.48 & 1.32 & 2.39 & 7.62 & 8.67 & 0.61 & 1.81 \\
\hline Species richness & 108 & 74 & 65 & 54 & 46 & 57 & 37 & 30 \\
\hline Total families & 29 & 25 & 20 & 21 & 18 & 20 & 15 & 11 \\
\hline Total abundance & 12.881 & 2.326 & 680 & 3.886 & 774 & 1.326 & 2.620 & 1.269 \\
\hline Relative abundance $(\%)$ & 100 & 18.05 & 5.27 & 30.17 & 6.0 & 10.29 & 20.34 & 9.85 \\
\hline
\end{tabular}

1= Majahua, $2=$ Tlacopanocha, $3=$ Parque de la Reina, 4= Muelle, $5=$ Manzanillo, $6=$ La Angosta and 7= Pie de la Cuesta 
Octomarginula natlandi

Diodora digueti

D. inaequalis

D. saturnalis

Fissurella asperella

$F$. decemcostata

$F$. deroyae

F. gemmata

F. macrotrema

10) F. microtrema

11) F. nigrocincta

12) F. obscura

13) F. rubropicta

14) Lottia acutepex

15) L. mitella

16) L. mesoleuca

17) L. pediculus

18) L. fascicularis

19) Patelloida semirubida

20) Calliostoma aequisculptum

21) Tegula globulus

22) T. panamensis

23) Monilea patricia

24) Arene hindsiana

25) Uvanilla unguis

26) Eulithidium perforatum

27) Nerita scabricosta

28) N. funiculata

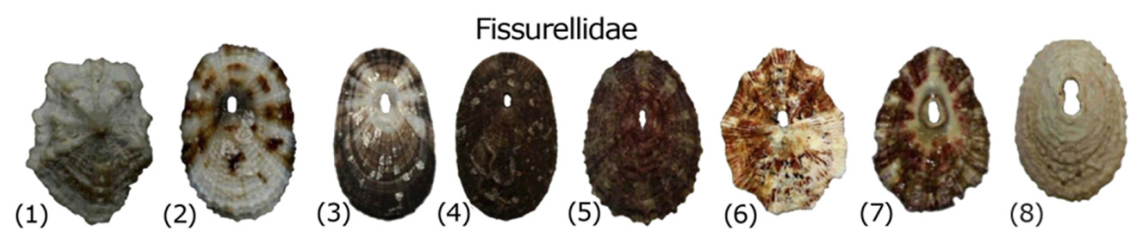

(8)

(1)

(2)

(3)
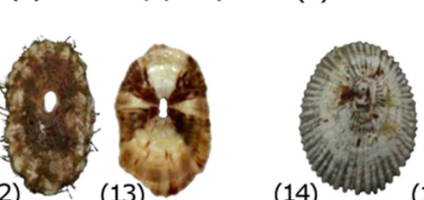

ottiidae

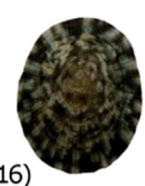

14) Mudu (15)
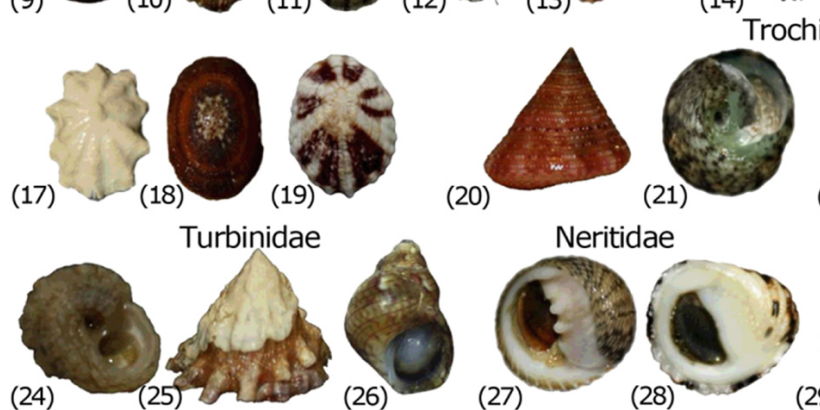

Epitoniidae

Rissoidae Cerithiida

Planaxida
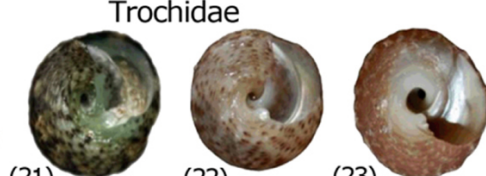

(23)

Littorinidae
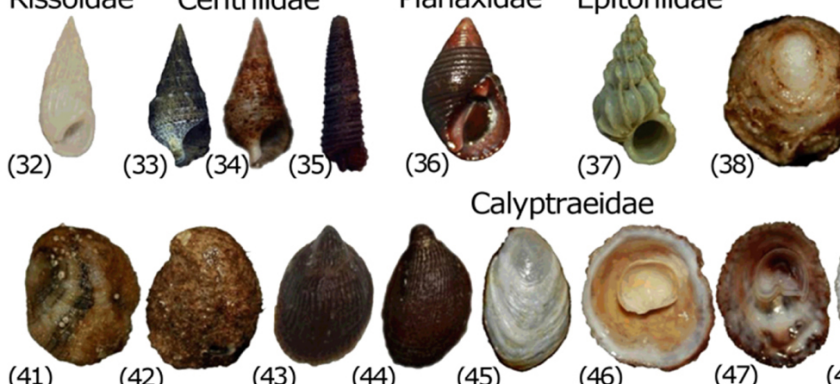

(30) (31)

Hipponicidae

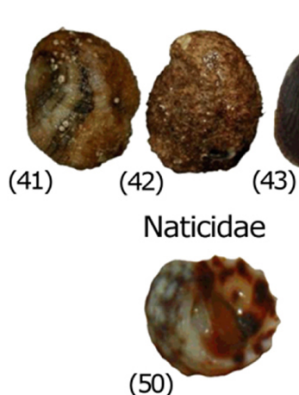

(50)

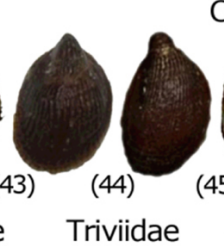

Calyptraeidae
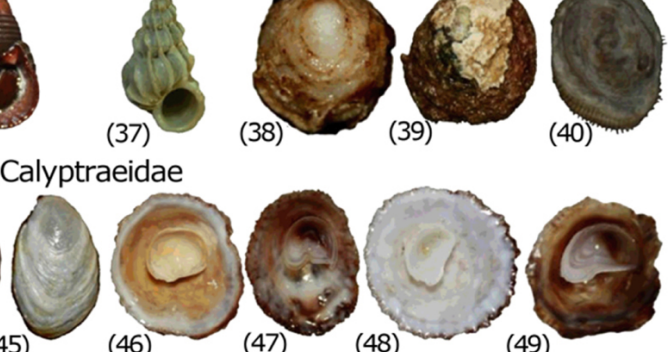

29) Echinolittorina aspera

30) E. peruviana

31) E. modesta

32) Rissoina stricta

33) Cerithium menkei

34) C. gemmatum

35) Seila assimilata

36) Planaxis obsoletus

37) Epitonium cookeanum

38) Hipponix panamensis

39) H. delicatus

40) Pilosabia trigona

41) Bostrycapulus acueleatus

42) Crepidula excavata

43) C. incurva

44) C. onyx

45) C. striolata

46) Crucibullum subactum

47) C. umbrela

48) C. cyclopium

49) C. scutellatum

50) Natica grayi

51) Niveria pacifica

52) Macrocypraea cervinetta

53) Mauritia arabicula

54) Jenneria pustulata

55) Monoplex vestitus

Picture: LECYS-UAGro 
56) Aspella hastula

57) Acanthais brevidentata

58) Vasula speciosa

59) Acanthais triangularis

60) Murexsul zeteki

61) Hexaplex princeps

62) Plicopurpura columellaris

63) P. pansa

64) Stramonita biserialis

65) Trachypollia lugubris

66) Vasula melones

67) Vitularia salebrosa

68) Coralliophila parva

69) Gemophos sanguinolentus

70) Engina tabogaensis

71) E.pulcra

72) Columbella auromexicana

73) C. fuscata

74) C. major

75) C. sonsonatensis

76) Cosmioconcha palmeri

77) Anachis nigrofusca

78) A. spadicea

79) Decipifus lyrta

80) Mitrella ocellata

81) M. xenia

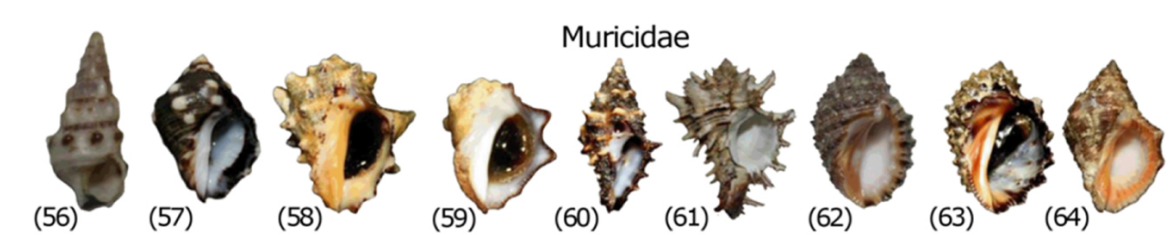

Buccinidae
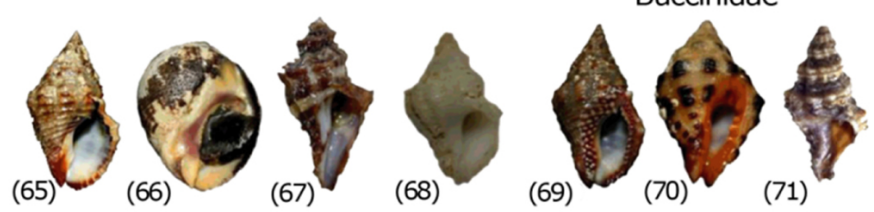

Columbellidae

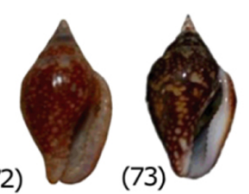

(7)
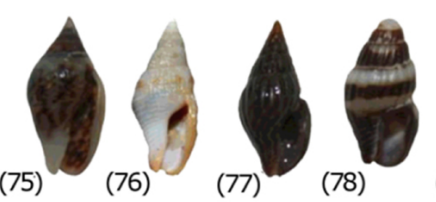

(79)

(71)
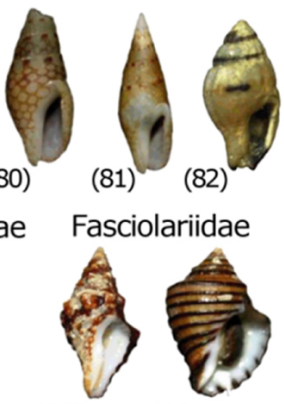

(89)

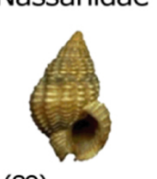

(85)

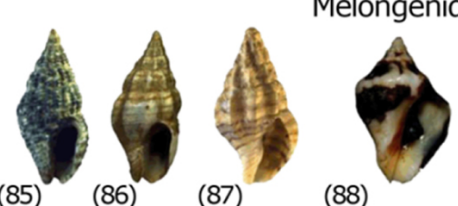

Mitridae Cancellariidae
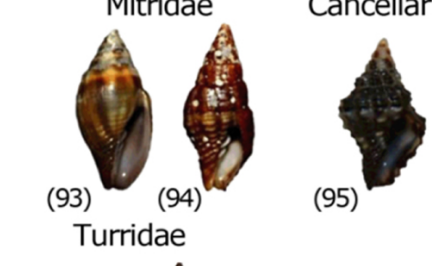

(95)

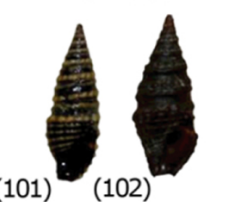

Subclass

Pulmonata

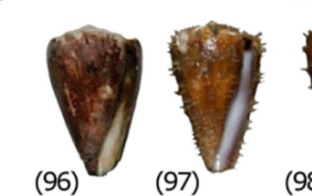

(91)

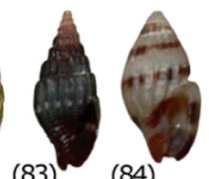

Olividae

Fasciolariidae

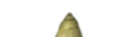

$\theta$

(92)

Conidae

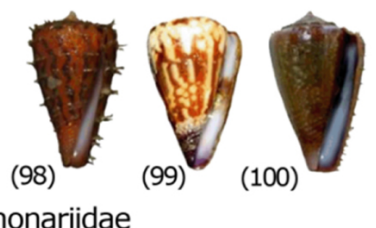

Siphonariidae

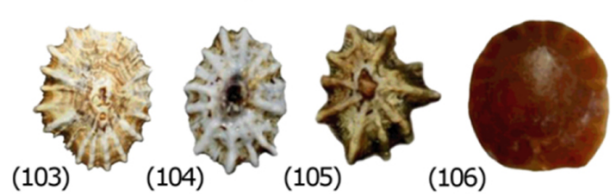

82) Parvanachis dalli

83) P. guerreorensis

84) P. pygmaea

85) Gilptanachis hilli

86) Anachis gaskoini

87) A. scalarina

88) Melongena patula

89) Nasarius gayii

90) Leucozonia cerata

91) Opeatostoma pseudodon

92) Olivella dama

93) Mitra tristis

94) M. rubicola

95) Admete viridula

96) Conus brunneus

97) C. gladiator

98) C. princeps

99) С. пих

100) C. purpurascens

101) Pilsbryspira garciacubasi

102) Crassispira cerithoidea

103) Siphonaria gigas

104) S. maura

105) S. palmata

106) Williamia peltoides

Picture: LECYS-UAGro

Figure 3. Species ridmess of the Pulmonata and Prosobranchia subdasses, associated to the rocky intertidal zone of the MPR 32, Guerrero, Mexico/ Riqueza de especies de las subclases Pulmonata y Prosobranchia, asociadas a la zona intermareal rocosa del la RMP 32, Guerrero, México 
The higher relative abundance per site was registered in Parque de la Reina (30.17\%), followed by La Angosta $(20.34 \%)$. Sites that showed lower values in relative abundance are Muelle (6\%) and Tlacopanocha (5.27\%).

In the MPR 32, the species registered with high relative abundance was Acanthais triangularis (Blainville, 1832) (12.74\%), followed by Tegula globulus (Carpenter, 1857) $(8.75 \%)$, Mitrella ocellata (Gmelin, 1791) (7.77\%), Stramonita biserialis (Blainville, 1832) (6\%), Siphonaria maura Sowerby I, 1825 (6.08\%) and Columbella fuscata G. B. Sowerby I, 1832 (5.38\%). 21 species had greater or equal to $1 \%$ relative abundance. Together these 21 species accounted for $86.83 \%$ of the total abundance found in the MRP 32 . The $0.31 \%$ of the total abundance is represented by 28 species; these species are represented by an organism. Only 5 sites these species (7 in Majahua, 10 in Tlacopanocha, 4 in Parque de la Reina, 3 in Muelle and 4 in Manzanillo) were found (Table 2).

The most abundant species recorded in Majahua were $S$. maura (24.24\%) and Siphonaria palmata Carpenter, 1857 (12.98\%) in Tlacopanocha, T. globulus (29.26\%) and Echinolittorina aspera (Philippi 1846) (10.29\%), in Parque de la Reina, T. globulus (21.69\%) and A. triangularis (18.63\%), in Muelle, Anachis nigrofusca Carpenter, 1857 (0.33\%) and C. fuscata (9.30\%), in Manzanillo, Parvanachis dalli (Bartsch, 1931) (18,25\%) and Bostrycapulus acueleatus (Gmelin, 1791) (6.48\%), In La Angosta, A. triangularis (17.25\%) and A. nigrofusca with (16.48\%) and in Pie de la Cuesta, Fissurella nigrocincta Carpenter, 1856(17.73\%) and Fissurella gemmata Menke, 1847 (12.13\%).

12 families of the subclass Prosobranchia presented broad distribution, 8 with frequent distribution and 8 with restricted distribution. The Siphonaridae family, which belongs to the subclass Pulmonata also found with broad distribution. Subclass Prosobranchia, 23 species were found with broad distribution, with frequent distribution 27 and 54 with restricted distribution. In the subclass Pulmonata 2 species were found with broad distribution, 1 species with frequent distribution and 1 with restricted distribution.

Macrocyprea cervinetta (Kiener, 1843) had the greatest size in length with $84.48 \mathrm{~mm}(\mathrm{~min}=7.97 ; 37.82 \pm 24.12)$, followed by Leucozonia cerata (Wood, 1828) to $69.13 \mathrm{~mm}$ $(\min =07.9 ; 23.78 \pm 10.66)$ and Crucibulum umbrella (Deshayes, 1830$)$ to $65.85 \mathrm{~mm}(\mathrm{~min}=8.39 ; 38.29 \pm 10.99)$. The species that presented the smallest sizes on in length were Natica grayi (Philippi, 1852) with $2.32 \mathrm{~mm}$ and Eulithidium perforatum (Philippi, 1848) with $3.12 \mathrm{~mm}$ (Table 2).

The value of diversity index H' for the MPR 32, was 4.87 bits individual $^{-1}$, while the value of J' was 0.72 (Table 4).
Table 4. Estimated values of $\mathrm{H}^{\prime}$ and J' for the RMP 32, and for each sampling site / Valores de 'H' y J' estimado para el MPR 32 , y para cada sitio de muestreo

\begin{tabular}{lcc}
\hline Sampling site & $\mathrm{H}^{\prime}$ & $\mathbf{J}^{\prime}$ \\
\hline Majahua & 4.309 & 0.687 \\
Tlacopanocha & 4.479 & 0.736 \\
Parque de la Reina & 3.672 & 0.641 \\
Muelle & 4.285 & 0.776 \\
Manzanillo & 4.665 & 0.807 \\
La Angosta & 3.616 & 0.689 \\
Pie de la Cuesta & 3.659 & 0.761 \\
General & 4.873 & 0.720 \\
\hline
\end{tabular}

\section{DiscuSSION}

The Gastropoda class is well represented in the rocky intertidal zone of the MRP 32. The species richness of the Gastropoda Class along the Mexican Pacific, outside the state of Guerrero, is lower compared to the results of this research (Ortíz-Arellano \& Flores-Campaña 2008, Ríos-Jara et al. 2009, Landa-Jaime et al. 2013, Flores-Rodríguez et al. 2014), as well as in other sites located in the state of Guerrero (Flores-Rodríguez et al. 2012, Flores-Garza et al. 2010, 2011, Torreblanca 2010, 2012, Torreblanca-Ramírez et al. 2012). Only Salcedo et al. (1988) reported 118 species of subclass Prosobranchia, but they sampled in the intertidal and subtidal sites with sandy and rocky substrate.

The species richness corresponds to that expected in the rocky intertidal zone located in a tropical area. Differences in species richness from the rocky intertidal zone of the state of Guerrero, is attributed to greater sampling effort, the characteristics of easy access to the sites, and careful sampling of the area. Furthermore, sampling was conducted at sites that vary in the type of substrate and wave exposure.

The highest species richness was found on sites that have low stability of the substrate. Sites that have stable substrate and the impact of the waves is direct had lower species richness. The relationship between species richness, diversity and complexity of the substrate has been treated by classical works such as Brusca (1980), which mentions that there is a direct correlation between the stability of the beaches and the type of species. Spight (1977) found a higher species richness in substrates formed by pebbles, compared with cliffs. FloresRodríguez et al. (2012), analyzing the biodiversity of molluscs distributed in 9 sites on the coast of the state of Guerrero reported that species richness is associated with habitat stability and intensity of the waves. Unstable substrates formed by 
boulders and with less intensity of the waves, showed higher species richness.

Fissurellidae, Lottiidae, Muricidae, Columbellidae and Calyptraeidae were reported as families with higher species richness by Flores-Rodríguez et al. (2014), Flores-Garza et al. (2010), Torreblanca (2010), Flores-Garza et al. (2011), Torreblanca et al. (2012) and Torreblanca-Ramírez et al. (2012). The same result was found in this research. Moreover, in this study, family Siphonaridae subclass Pulmonata is reported as a family well represented in the MPR 32.

Muricidae and Columbellidae was determined as the most abundant families in the rocky intertidal of the MPR 32. Similar results are reported by Torreblanca et al. (2012) and Torreblanca-Ramírez et al. (2012), to Acapulco. Flores (2004), for the state of Guerrero reported to Lottiidae and Siphonariidae families as the most abundant. Furthermore, this research shows for the first time the Trochidae family as one of the most abundant for the rocky intertidal zone of the MPR 32 for the first time.

The subclass Prosobranchia subclass, families Fissurellidae, Lottiidae, Calyptraeidae, Muricidae and Columbellidae and Pulmonata subclass the Siphonaridae family, were identified as the best represented in species richness, abundance and distribution so they are considered as representative Families for the rocky intertidal zone of the MPR 32.

Species that this investigation found as the most abundant, are similar to those reported by other research conducted in the MPR 32 (Flores-Garza et al. 2010, 2011; Torreblanca et al. 2012 and Torreblanca-Ramírez et al. 2012). Furthermore, the present investigation reports $F$. gemmata, F. nigrocincta, Lottia mesoleuca (Menke, 1851), Lottia pediculus (Philippi, 1846) and Plicopurpura pansa (Gould, 1853).

The abundances for 28 species were represented by a single organism, and 6 species for 2, these species are considered rare for the rocky intertidal zone of the MPR 32. The rare species found only in the sites whose characteristic wave exposure is low to medium. Rare species were not found at stations with high exposure to waves.

Rare species are those having less resistance to environmental stress. Alcolado (2001) mentions that the higher correspondence of the values of environmental variables, with ranges favorable for physiological life, the greater the number of potential species that occurs in a given habitat and the less frequent and smaller the fluctuations in these variables, the greater the opportunities for each of the species to exploit to the maximum the available resources and increase their populations. However, molluscs species richness or heterogeneity, comparatively reflect the degree of stress, equity and the level of environmental forecasting. Therefore, the ecology is based on the identity of the species or its dominance (generalist species, widely distributed, abundant populations of larger sizes and greater support for environmental variations), and sometimes in the census of absent species (species specialists, more sensitive, their populations are smaller, have less resistance to environmental variations).

In relation to geographical distribution of species, $L$. mesoleuca, L. pediculus, L. fascicularis, C. umbrella, V. speciosa, A. triangularis, P. pansa, S. biserialis, T. lugubris, Engina tabogaensis Bartsch, 1931, C. fuscata, A. nigrofusca, M. ocellata, M. xenia, P. dalli, S. maura and S. palmata are the representative species of the rocky intertidal zone MPR 32 , these species were present at the 7 sites.

Species that have been reported with the greater size in the MPR 32 are C. umbrella and M. cervinetta (Flores-Garza et al. 2011, Torreblanca et al. 2012 and Torreblanca-Ramírez et al. 2012). This research found similar results, and also reports L. cerata as a species with larger size. $N$. grayi and Jenneria pustulata (Lightfoot, 1786) have been reported in the MPR 32 as the smaller species (Flores-Garza et al. 2011, Torreblanca et al. 2012, Torreblanca-Ramírez et al. 2012). This research also found $N$. grayi as one species with the smallest size in length.

The values of diversity and eveness for molluscs, which have been reported by other studies conducted in sites located in the MPR 32 are high and similar to those found in the present study (Flores-Garza et al. 2011, Torreblanca et al. 2012 and Torreblanca-Ramírez et al. 2012). Finally, the values of diversity and uniformity found in the study area correspond to that of a tropical zone and are evidence that the region studied is a highly diverse area and it corresponds to benthic ecosystems with high stability.

\section{ACKNOWLEDGMENTS}

We acknowledge the Council for Science and Technology, Mexico (CONACYT), for the support provided through a postgraduate scholarship.

\section{LITERATURE CITED}

Alcolado MP. 2001. Diversidad y bioindicación ambiental en el mar. Instituto de Oceanología, Agencia de Medio Ambiente, Ministerio de Ciencia, Tecnología y Medio Ambiente (CITMA), Ciudad de La Habana. <http://oceanologia. redciencia.cu/articulos/articulo08.pdf $>$

Arriaga L, E Vázquez, J González, R Jiménez, E Muñoz \& V Aguilar. 1998. Regiones prioritarias y planeación para la conservación de la biodiversidad, en Capital natural de México. Estado de conservación y tendencias de cambio, pp. 433-457. CONABIO, México. 
Barba-Marino F, P Flores-Rodríguez, R Flores-Garza, S García-Ibáñez \& DG Arana-Salvador. 2010. Biodiversidad y zonificación de la comunidad de moluscos, que habita el sustrato rocoso en dos sitios con distinta acción del oleaje, en la Isla 'La Roqueta' Acapulco, Guerrero, México. En: Rangel LJ, J Gamboa, SL Arriaga \& WM Contreras (eds). Perspectiva en malacología mexicana, pp. 19-44. Universidad Juárez Autónoma de Tabasco, Villahermosa.

Brusca RC. 1980. Common intertidal invertebrates of the Gulf of California, 513 pp. University of Arizona Press, Tucson.

Carranza-Edwards A, A Márquez-García \& E Morales de la Garza. 1986. Estudio de sedimentos de la plataforma continental del Estado de Guerrero y su importancia dentro de los recursos minerales del mar. Anales del Instituto de Ciencias del Mar y Limnología, Universidad Nacional Autónoma de México 13(3): 241-262.

Delgado H. 1989. Estudio sistemático y aspectos ecológicos de gasterópodos de la facie rocosa de la bahía de Acapulco, Guerrero, México. Tesis de Licenciatura, Escuela Superior de Ecología Marina, Universidad Autónoma de Guerrero, Acapulco, 97 pp.

Esqueda MC, E Ríos-Jara, JE Michel-Morfín \& V LandaJaime. 2000. The vertical distribution and abundance of gastropods and bivalves from rocky beaches of Cuastecomate Bay, Jalisco. Revista de Biología Tropical 48: 765-775.

Flores P. 2004. Estructura de la comunidad de moluscos del mesolitoral superior en las playas de facie rocosa del Estado de Guerrero, México. Tesis Doctoral, Facultad de Ciencias Biológicas, Universidad Autónoma de Nuevo León, San Nicolás de los Garza, 207 pp.

Flores-Garza R, C Torreblanca-Ramírez, P FloresRodríguez, S García-Ibáñez \& L Galeana-Rebolledo. 2010. Riqueza y análisis de la comunidad malacológica en el mesolitoral rocoso de la playa Tlacopanocha, Acapulco, Guerrero. En: Rangel LJ, J Gamboa, S Arriaga \& W Contreras (eds). Perspectivas en malacología mexicana, pp. 123-138, Universidad Juárez Autónoma de Tabasco, Villahermosa.

Flores-Garza R, C Torreblanca-Ramírez, P FloresRodríguez, S García-Ibáñez, L Galeana-Rebolledo, A Valdés-González \& A Rojas-Herrera. 2011. Mollusc community from a rocky intertidal zone in Acapulco, Mexico. Biodiversity 12(3): 144-153.

Flores-Garza R, L Galeana-Rebolledo, A Reyes-Gómez, S García-Ibáñez, C Torreblanca-Ramírez, P FloresRodríguez \& A Valdés. 2012. Polyplacophora species richness, composition and distribution of its community associated with the intertidal rocky substrate in the marine priority region No. 32 in Guerrero, Mexico. Open Journal of Ecology 2: 192-201. <http://www.scirp.org/journal/ PaperInformation. aspx?paperID=24567\#.U0bdzvl5OX4>

Flores-Rodríguez P, R Flores-Garza, S García-Ibáñez \& A Valdés-González. 2003. Riqueza y diversidad de la malacofauna del mesolitoral rocoso de la Isla la Roqueta, Acapulco, Guerrero, México. Ciencia, Revista de Investigación Científica 11: 5-14.
Flores-Rodríguez P, R Flores-Garza, S García-Ibáñez \& A Valdés-González. 2007. Variación en la diversidad malacológica del mesolitoral rocoso en Playa Troncones La Unión, Guerrero, México. Revista de Biología Tropical 55(34): $867-887$.

Flores-Rodríguez P, F Barba-Marino, R Flores-Garza, $S$ García-Ibáñez \& DG Arana-Salvador. 2010. Análisis de la comunidad de moluscos del mesolitoral rocoso en playa Corralero, Oaxaca, México. En: Rangel LJ, J Gamboa, SL Arriaga \& WM Contreras (eds). Perspectiva en malacología mexicana, pp. 77-88, Universidad Juárez Autónoma de Tabasco, Villahermosa.

Flores-Rodríguez P, R Flores-Garza, S García-Ibáñez, A Valdés-González, J Violante-González, CE Santiago, L Galeana-Rebolledo \& C Torreblanca-Ramírez. 2012. Mollusk species richness on the rocky shores of the State of Guerrero, Mexico, as affected by rains and their geographical distribution. Natural Resources 3: 248-260.

Flores-Rodríguez P, R Flores-Garza, S García-Ibáñez, C Torreblanca-Ramírez, L Galeana-Rebolledo \& E Santiago-Cortes. 2014. Mollusks of the rocky intertidal zone at three sites in Oaxaca, Mexico. Open Journal of Marine Science 4: 326-337. <http://dx.doi.org/10.4236/ ojms.2014.44029>

García A. 1994. Fauna malacológica de acompañamiento del caracol Purpura pansa Gould1853 en la zona mesolitoral de la isla Roqueta, Acapulco, Guerrero, México. Tesis de Licenciatura, Escuela Superior de Ecología Marina, Universidad Autónoma de Guerrero, Acapulco, 97 pp.

González-Villareal LM. 2005. Guía ilustrada de los gasterópodos marinos de la bahía de Tenacatita, Jalisco, México. Scientia-CUCBA 7(1): 1-84.

Holguín OE \& AC González. 1989. Moluscos de la franja costera del Estado de Oaxaca, México, 221 pp. Dirección de Bibliotecas y Publicaciones, Instituto Politécnico Nacional, Ciudad de México.

Holguín-Quiñones OE \& AC González-Pedraza. 1994 Moluscos de la franja costera de Michoacán, Colima y Jalisco, México, 133 pp. Dirección de Bibliotecas y Publicaciones, Instituto Politécnico Nacional, Ciudad de México.

Keen AM. 1971. Sea shells of tropical west America, 1064 pp. Stanford University Press, San Diego.

Landa-Jaime V \& J Arciniega-Flores. 1998. Macromoluscos bentónicos de fondos blandos de la plataforma continental de Jalisco y Colima, México. Ciencias Marinas 24: 155-167.

Landa-Jaime V, M Cruz-Urzua, JE Michel-Morfín, J Archiniega-Flores, R Flores-Vargas \& C Amezcua. 2007. Guía ilustrada para la identificación de moluscos intermareales y de arrecifes en la Bahía de Tenacatita, Jalisco. En: Ríos-Jara E, MC Esqueda-González \& CM Galván-Villas (eds). Estudios sobre la malacología y conquiliología en México, 286 pp. Universidad de Guadalajara, México. 
Landa-Jaime V, E Michel-Morfín, J Arciniega-Flores, S Castillo-Vargasmachuca \& M Saucedo-Lozano. 2013. Moluscos asociados al arrecife coralino de Tenacatita, Jalisco, en el Pacífico central de mexicano. Revisa Mexicana de Biodiversidad 84: 1121-1136.

López FA \& JR Urcuyo. 2009. Moluscos de Nicaragua II, Gastrópodos. 181 pp. Marena-Araucaria, UCA, Managua.

Mottana A, R Crespi \& G Liborio. 1980. Guía de minerales y rocas, 605 pp. Editorial Grijalbo, Barcelona.

Olabarría C \& C Vega. 2000. Extensión del ámbito geográfico de algunas especies de moluscos marinos del en Estado de Sinaloa, México. Anales del Instituto de Biología, Universidad Nacional Autónoma de México, Serie Zoológica 71(1): 93-98.

Ortíz-Arellano MA \& LM Flores-Campaña. 2008. Catálogo descriptivo e ilustrado de los moluscos de la zona intermareal de las islas de Navachiste, Sinaloa, México, 132 pp. Universidad Autónoma de Sinaloa, Gobierno del Estado de Sinaloa, Consejo Nacional de Ciencias y Tecnología, Mazatlán.

Reguero M \& A García-Cubas. 1989. Moluscos de la Plataforma Continental de Nayarit: Sistemática y ecología (cuatro campañas oceanográficas). Anales del Instituto de Ciencias del Mar y Limnología, Universidad Nacional Autónoma de México 16: 33-58.

Ríos-Jara E, M Pérez, L Lizárraga \& E Michel-Morfín. 1996. Nuevos registros de la plataforma continental de Jalisco y Colima, México. Ciencias Marinas 22(3): 347-359.

Ríos-Jara E, CM Navarro-Caravantes, CM Galván-Villa \& E Lopez-Uriarte. 2009. Bivalves and Gastropods of the Gulf of Tehuantepec, Mexico: A checklist of species with notes on their habitat and local distribution, Journal of Marine Biology 2009, Article ID 176801. <http://dx.doi.org/10.1155/2009/ $176801>$

Román R, FM Cruz \& AL Ibáñez. 1991. Observaciones ecológicas de los moluscos de la zona intermareal de la bahía de Chamela, Jalisco, México. Anales del Instituto de Biología, Universidad Nacional, Autónoma de México, Serie Zoología 62: $17-32$

Salcedo S, G Green, A Gamboa \& P Gómez. 1988. Inventario de macroalgas y macroinvertebrados bénticos, presentes en áreas rocosas de la región de Zihuatanejo, Guerrero, México. Anales del Instituto de Ciencias del Mar y Limnología, Universidad Nacional Autónoma de México 15: 73-96.

Skoglund C. 2002. Panamic province molluscan literature additions and changes from 1971 through 2001, III Gastropoda, 286 pp. The Festivus, San Diego.

Spight TM. 1977. Diversity of shallow-water gastropods communities on temperate and tropical beaches. American Naturalist 111: 1077-1097.
Tenorio JM, JK Tucker \& HW Chaney. 2012. The Families Conilithidae and Conidae The Cones of the Eastern Pacific, 112 pp. ConchBooks, Hackenheim.

Torreblanca C. 2010. Análisis de la diversidad y estructura de la comunidad de moluscos del mesolitoral rocoso de Acapulco, Gro. Tesis de Licenciatura, Unidad Académica de Ecología Marina, Universidad Autónoma de Guerrero, Acapulco, Guerrero, 216 pp.

Torreblanca C, R Flores, P Flores, S García, A Valdés \& L Galeana. 2012. Gastrópodos del intermareal rocoso en Tlacopanocha, Acapulco, México. Tlamati Sabiduria, Universidad Autónoma de Guerrero 4(1): 47-57.

Torreblanca-Ramírez C, R Flores-Garza, P FloresRodríguez, S García-Ibáñez \& L Galeana-Rebolledo. 2012. Riqueza, composición y diversidad de la comunidad de moluscos asociada al sustrato rocoso intermareal de playa Parque de la Reina, Acapulco, México. Revista de Biología Marina y Oceanografía 47(2): 283-294.

Torreblanca-Ramírez C, R Flores-Garza, JE MichelMorfin, JL Rosas-Acevedo, P Flores-Rodríguez \& S García-Ibáñez. 2014. New records for Gastropoda Class of species found in the rocky intertidal zone of the marine priority region 32, Guerrero, Mexico Impacts of US Reindustrialization on Chinese Manufacturing. Open Journal of Marine Science 4: 221-237. <http://dx.doi.org/10.4236/ ojms.2014.43021>

Tucker J \& M Tenorio. 2009. Systematic classification of recent and fossil Conoidean Gastropods, 296 pp. Hackenheim, ConchBooks.

Vega C, C Olabarria \& JL Carballo. 2008. Variación espaciotemporal de moluscos y macroalgas en sustratos rocosos intermareales en la bahía de Mazatlán. Ciencia y Mar 12(34): 3-16.

Villalpando E. 1986. Diversidad y zonación de moluscos de superficie rocosa, Isla Roqueta, Acapulco, Guerrero. Tesis de Licenciatura, Facultad de Ciencias, Universidad Nacional Autónoma de México, México, 150 pp.

Villarroel MM, A Magaña, B Gómez, O Del Río, J Lucio \& J Sánchez. 2000. Diversidad de moluscos en el litoral rocoso de Michoacán, México. Mexico 2: 54-63.

Wilkinson T, E Wiken, J Bezaury-Creel, T Hourigan, T Agardy, H Herrmann, L Janishevski, C Madden, L Morgan \& M Padilla. 2009. Ecorregiones marinas de América del Norte, 200 pp. Comisión para la Cooperación Ambiental, Montreal.

Zamorano P, NA Barrientos-Luján \& S Ramírez-Luna. 2008. Malacofauna del infralitoral rocoso de Agua Blanca, Santa Elena Cozoaltepec, Oaxaca. Ciencia y Mar 12(36): 19-33. 